\title{
Research on NLOS Channel Based on IEEE 802.15.3c Standard
}

\author{
Xiaolin Liang ${ }^{1, a}$, Hao Zhang ${ }^{1, b}$ and Tingting Lv ${ }^{1, c}$ \\ ${ }^{1}$ College of Information Science and Engineering, Ocean University of China, \\ Qingdao, 266100, China \\ axiaolin87liang@163.com, 'bhanghao@ouc.edu.cn, 'Ivtingting33@163.com
}

Keywords: NLOS identification, IEEE 802.15.3c, energy detector, $60 \mathrm{GHz}$

\begin{abstract}
The major problem of indoor localization is the presence of non-line-of-sight (NLOS) channels. In order to perform NLOS identification, a novel NLOS identification technique is proposed which is based on the multiply of the standard deviation, max-slope and max-gradient of the block using energy detector (ED). IEEE 802.15.3c $60 \mathrm{GHz}$ channel models are used as examples and the above statistics are explained in detail. The simplicity of proposed approach lies in ED-based algorithm. The LOS and NLOS of IEEE 802.15.3c channel models are used. Simulations results show the correct identification is better than with the multipath channel statistics based approach.
\end{abstract}

\section{Introduction}

The $60 \mathrm{GHz}$ has a great potential for accurate ranging and localization systems due to its very wide bandwidth and capability in resolving individual multipath components [1-3]. Therefore, TOA can be estimated with high accuracy for the $60 \mathrm{GHz}$ systems if the first arriving path has been identified precisely. One of the major challenges for localization systems is mitigation of NLOS effects. If the direct path between a fixed terminal and mobile terminal is being obstructed, TOA to the fixed terminal will be delayed, which introduces a positive bias. Using such NLOS TOA, the mobile terminal position may significantly degrade positioning accuracy.

The non-line of sight identification techniques have been discussed extensively in the literature, but mainly within the cellular network framework [4-10]. For example, in [7], the authors address the NLOS identification problems based on the multiple received signal strength measurements from Wi-Fi signals. The key to the approach is to exploit several statistical features of the RSS time series. Shimizu et al. [9] performed intensive measurements of path-loss and delay-profile characteristics of line-of-sight and non-line-of-sight environments in a suburban residential area. Based on their analysis, they found that the delay spread was dependent on distance, and the non-line-of-sight delay spread was found to be several times larger than that of the line-of-sight case. The skewness of delay spread for the non-line-of-sight cases ranged from 80 to 200ns, which was an order of magnitude larger than that of the line-of-sight case.

In this paper, a new NLOS identification approach is proposed for the $60 \mathrm{GHz}$ signal, which is based on the standard deviation, max-slope, max-gradient of the energy block using ED. Firstly, we use ED-based algorithm for TOA estimation. Secondly, we characterize the standard deviation, max-slope, max-gradient of the block. Finally, we use a threshold test for NLOS identification.

The remainder of this paper is organized as follows. Section 2 describes signal and channel model. Section 3 describes NLOS identification approach and Section 4 presents results of numerical simulations. The concluding remarks are given in section 5 .

\section{System Model}

The $60 \mathrm{GHz}$ signals have a very short duration and which can be expressed as:

$$
s(t)=\sum_{-\infty}^{\infty} p\left(t-j T_{s}-C_{j} T_{c}-a_{j} \varepsilon\right)
$$


where $T_{s}$ is symbol time. The time hopping code represented by $C$ is a pseudorandom integer-valued sequence which is unique for each user to limit multiple access interference, and $T_{c}$ is chip time. The PPM time shift is $\varepsilon$ so that if $a_{j}$ is 1 , the signal is shifted in time by $\varepsilon$, while if $a_{j}$ is 0 , there is no shift. In this paper a Gaussian pulse is employed which is multiplied by the carrier signal to give:

$$
p(t)=\frac{\sqrt{2}}{\alpha} \exp \left(-2 \pi \frac{t^{2}}{\alpha^{2}}\right) \cos \left(2 \pi f_{c} t\right)
$$

where $\alpha$ is shape factor and $f_{c}$ is carrier frequency which here is $f_{c}=60 \mathrm{GHz}$. A smaller shape factor results in a shorter duration pulse and a larger bandwidth. The received signal can be written as:

$$
r(t)=\sum_{n=1}^{N} \alpha_{n} p\left(t-\tau_{n}\right)+n(t)=s(t) * h(t)+n(t)
$$

where $N$ is the number of received multipath components, $\alpha_{n}$ and $\tau_{n}$ denotes amplitude and delay of the $n t h$ path respectively, $n(t)$ is the AWGN with zero mean and two sided power spectral density $\mathrm{N}_{0} / 2$, and $h(t)$ is the channel impulse response which can be expressed as:

$$
h(t, \theta)=\sum_{k=1}^{K} \sum_{l=1}^{L_{k}} \mu_{k l} \delta\left(t-T_{k}-\tau_{k l}\right) \delta\left(\theta-\theta_{k}-\omega_{k l}\right)
$$

where $\delta($.$) is the dirac-delta function, K$ is the number of clusters, $L_{k}$ is the number of rays in the $k^{\text {th }}$ cluster, and $\mu_{k l}, \tau_{k l}$ and $\omega_{k l}$ denote complex amplitude, delay and azimuth of the $k^{\text {th }}$ ray of the $l^{\text {th }}$ cluster, respectively. Similarly, $T_{k}$ and $\theta_{k}$ represent delay and mean AOA of the $k^{\text {th }}$ cluster.

After amplifier, the received signals are squared, and then input to an integrator with integration period $T b$. The integrator outputs can be expressed as:

$$
z[n]=\sum_{i=1}^{N} \int_{(i-1) T_{f}+\left(c_{j}+n-1\right) T b}^{(i-1) T_{f}+\left(c_{j}+n\right) T b} r^{2}(t) d t
$$

where $n \in\{1,2, . ., N\}$ denotes the sample index with respect to the starting point of integration period and $N$ is the number of the pulses per symbol.

\section{NLOS Identification}

In this paper, we distinguish NLOS scenarios by exploiting the statistics of the ED-based received signal. The standard deviation, max-slope, max-gradient of energy block is used to identify NLOS scenarios. The slope of the energy values is considered as a measure. These values are divided into ( $N-M+1)$ groups, with $M$ values in each group. Slope for each group is calculated using a least squares line-fit. Slope can then be expressed as:

$$
S=\underset{1 \leq n \leq N-M+1}{\operatorname{slope}}\{\operatorname{linefit}(z[n], z[n+1], \ldots \ldots, z[n+M-1])\}
$$

The standard deviation is a widely used measure of variability. It shows how much variation or "dispersion" there is from the average which is given by:

$$
D=\sqrt{\frac{\sum_{i=1}^{N}\left(x_{i}-\mu_{x}\right)^{2}}{N-1}}
$$

where $\mu_{x}$ is the mean value.

The gradient is a generalization of the usual concept of derivative of a function in one dimension to a function in several dimensions. Similarly to the usual derivative, the gradient represents the slope of the tangent of the graph of the function. The received samples $z[n] n \in\left\{1,2, \cdots, N_{b}\right\}$ can be expressed as $N_{b} * 1$ dimensional matrix referred as $\mathbf{U}$. The GRADIENT function provided by the MATLAB is applied to calculate the gradient of the energy values.

The multiply of the standard deviation, max-slope and max-gradient of the energy block can be obtained for NLOS identification, which can be expressed as: 


$$
\text { Joint Metric }=D^{*} \text { MaxiS } * \text { MaxiG }
$$

In order to examine characteristics of the multiply, CM1 and CM2 channel models from IEEE 802.15.3c standard are employed. For each SNR value, 1000 channel realizations are generated and sampled at $f_{c}=1 \cdot e^{10} \mathrm{~Hz}$. The other system parameters are $T_{f}=200 n s T_{c}=1 n s \quad T b=4 n s$ and $N=1$. Each realization has a TOA uniformly distributed within $\left(0-T_{f}\right)$. Here SNR ranges from 10 to 40dB, CM1 and CM2 are employed. Here 31*1000 samples are got for each channel model. The relationship between Joint Metric and SNR are shown in Fig. 1.

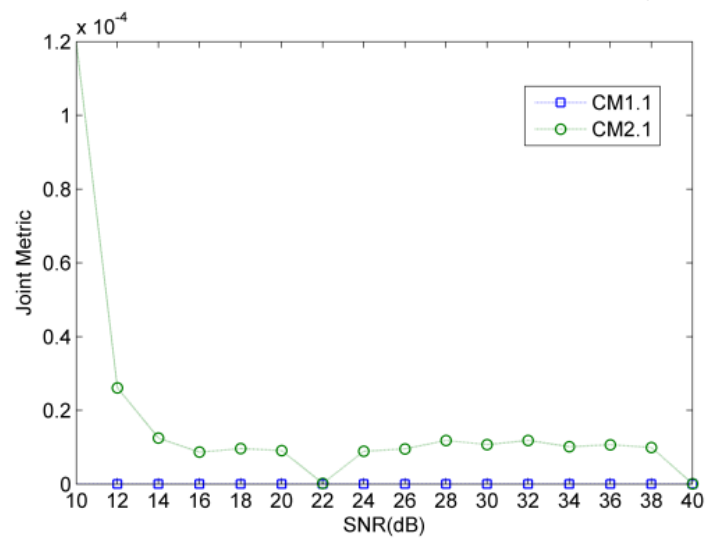

(1) $\mathrm{TX}=360^{\circ}$

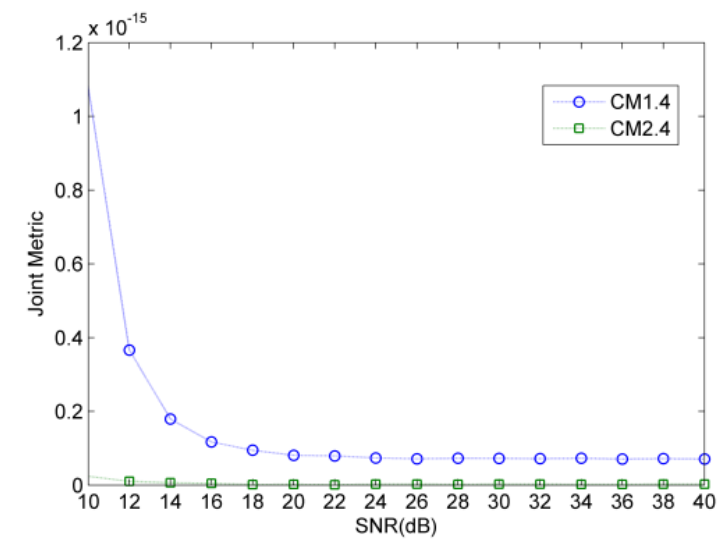

(2) $\mathrm{TX}<360^{\circ}$

Fig. 1. Joint metric with respect to SNR

These results show Joint Metric is monotonic with respect to SNR in both LOS and NLOS environments. It was found that maximum in LOS is less than minimum for a NLOS when the transmit antenna is omnidirectional $\left(T X=360^{\circ}\right)$. Conversely, minimum in LOS is larger than maximum in NLOS when the transmit antenna is not omnidirectional, which corresponds to channels $\left(T X<360^{\circ}\right)$. Note that for all channels $R X=15^{\circ}$. These results suggest the following method:

$$
\Theta=\alpha\left\{\begin{array}{l}
\left\{\begin{array}{l}
<\alpha_{M S} \Rightarrow \text { LOS } \\
>\alpha_{M S} \Rightarrow N L O S
\end{array} \quad T X=360^{\circ}\right. \\
\left\{\begin{array}{l}
>\alpha_{M S} \Rightarrow \text { LOS } \\
<\alpha_{M S} \Rightarrow N L O S
\end{array} \quad T X<360^{\circ}\right.
\end{array}\right.
$$

where $\alpha_{M S}$ is threshold which is chosen to identify NLOS, $T X, R X$ is the beam-width of measured transmitter and receiver antenna respectively. It can be shown as follows:

$$
\alpha_{M S}=\min \left\{\begin{array}{l}
\text { mean }\left[\text { find }\left(P\left((\text { Joint Metric })<\left.\Theta_{1}\right|_{A}\right) \geq \Phi\right)\right] \\
\text { mean }\left[\text { find }\left(P\left((\text { Joint Metric })<\left.\Theta_{2}\right|_{B}\right) \geq \Phi\right)\right]
\end{array}\right\}
$$

where $\Theta_{1}$ and $\Theta_{2}$ are threshold which meet the condition $P(M S>\Theta) \geq \Phi$ where SNR is upper and lower limits. $\Phi$ is the probability value which is required for choosing suitable threshold for NLOS identification. Without loss of generality, here $\Theta$ is set to be 0.85 .

In order to verify the effectiveness of NLOS identification algorithm, 1000 channel realizations were generated when SNR is from 10 to $40 \mathrm{~dB}$ in IEEE 802.15.3c channel and the other system parameters as above. The accuracy for identifying NLOS is calculated for each channel model. As shown in Table 1, the accuracy of NLOS identification is higher than any other identification algorithms based on ED such as in [11], the accuracy is only around $60 \%$ while the accuracy of the proposed algorithm over $80 \%$ for the most channel model. 
Table 2. Accuracy (\%) for NLOS Identification

\begin{tabular}{ccccccccccc}
\hline CM(dB) & 16 & 18 & 20 & 22 & 24 & 26 & 28 & 30 & 32 & 34 \\
\hline 1.1 & 92.6 & 92.9 & 92.9 & 93.7 & 93.3 & 93.7 & 93.5 & 93.6 & 93.2 & 93.6 \\
2.1 & 60.8 & 59.1 & 59.2 & 58.2 & 57.0 & 58.3 & 57.8 & 57.5 & 57.5 & 58.2 \\
1.2 & 100 & 100 & 100 & 99.9 & 98.8 & 98.1 & 98.7 & 99.1 & 99.0 & 98.7 \\
2.2 & 79.4 & 81.4 & 82.2 & 82.0 & 82.2 & 82.8 & 83.1 & 83.4 & 82.6 & 82.3 \\
1.3 & 100 & 100 & 100 & 100 & 100 & 100 & 100 & 100 & 100 & 100 \\
2.3 & 83.4 & 84.9 & 85.6 & 86.6 & 85.8 & 86.4 & 86.6 & 86.9 & 86.5 & 86.0 \\
1.4 & 100 & 100 & 100 & 100 & 100 & 100 & 100 & 100 & 100 & 100 \\
2.4 & 83.4 & 83.9 & 84.6 & 85.6 & 85.8 & 86.4 & 86.6 & 86.9 & 86.5 & 86.0 \\
\hline
\end{tabular}

\section{Conclusion}

In this paper, a novel approach to deal with NLOS propagation is proposed which is based on the multiply of the standard deviation, max-slope and max-gradient of the energy block of the received signal using energy detector. In order to verify the effectiveness and practicality of the algorithm, so we make a lot of simulations using IEEE 802.15.3c channel models. The CM1.1, CM1.2, CM1.3, CM1.4 (residential LOS) and CM2.1, CM2.2, CM2.3, CM2.4 (residential NLOS) channel models from IEEE 802.15.3c standard are employed. Results show that joint parameter can identify LOS and NLOS environments so long as threshold can be fixed bitterly.

\section{Acknowledgments}

This work was supported by the Nature Science Foundation of China under Grant No. 60902005, the International Science and Technology Cooperation Projects of Qingdao under Grant No. 12-1-4-137-hz, the Nature Science Foundation of China under Grant No. 61301139, the Nature Science Foundation of Shandong Province under Grant No. ZR2014FL014, the Science and Technology Project in Colleges and Universities of Shandong Province under Grant No.J14LN53, the Project of Basic Research Application of Qingdao City under Grant No. 14-2-4-37-jch, and the Project of Basic Research Application of Qingdao City under Grant No. 14-2-4-83-jch.

\section{Reference}

[1] R.C. Daniels, J.N. Murdock, T.S. Rappaport and R.W. Heath: IEEE Microw. Mag. Vol. 11 (2010), p. 44.

[2] Z. Pi and F. Khan: IEEE Commun. Mag. Vol. 49 (2011), p. 101.

[3] N. Chahat, G. Valerio, M. Zhadobov and R. Sauleau: IEEE Trans. Antennas Propagat. Vol. 61 (2013), p. 1876.

[4] K. Yu and E, Dutkiewicz: IEEE Trans Aerosp Electron Syst, Vol. 49 (2013), p. 1438.

[5] S. Tian, L. Zhao and G. Li: Math Probl Eng, Vol. 2014 (2014), doi.10.1155/2014/963418.

[6] Z. Wang, S. Zekavat and A. Reza: IEEE T Mobile Comput, Vol. 11 (2012), p. 2047-2059.

[7] Z. Xiao, H. Wen, A. Markham, N. Trigoni, P. Blunsom and J. Frolik: IEEE Tran Wirel Commun, Vol. 14 (2015), p. 1689.

[8]S. Wu, J. Li and S. Liu: Int J Commun Syst, Vol. 27 (2014), p. 2156.

[9] S. Venkatesh and R. M. Buehrer: IET Microw Antenna P, Vol. 1 (2007), p.1120.

[10] I. Güvenç, C. C. Chong, F. Watanabe and H. Inamura: EURASIP J Adv Sig Pro, Vol. 2008 (2008).

[11] S. H. WU, Q. Y. Zhang, N. T. Zhang: Vol. 30, (2008), pp. 2541. 